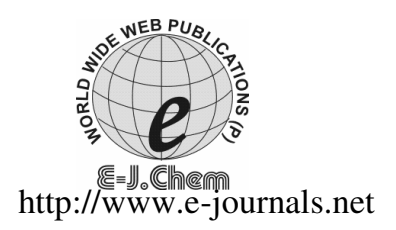

ISSN: 0973-4945; CODEN ECJHAO
E-Journal of Chemistry
2010, 7(4), 1320-1325

\title{
Synthesis of Some 2-Thioxo-imidazolidin-4-one Derivatives and its Antimicrobial Activity
}

\author{
A. JAMAL ABDUL NASSER ${ }^{\S *}$, A.IDHAYADHULLA ${ }^{\S}$, \\ R.SURENDRA KUMAR ${ }^{\S}$ and J.SELVIN \\ ${ }^{\S}$ P.G \& Research Department of Chemistry, \\ Jamal Mohamed College, Tiruchirapalli-620020, Tamilnadu, India. \\ Department of Microbiology, Bharathidasan University, \\ Tiruchirapalli-620024, Tamilnadu, India. \\ idhaya25chem@yahoo.co.in
}

Received 26 March 2009; Revised 8 June 2009; Accepted 2 August 2009

\begin{abstract}
Series of newly prepared 3-\{[2,6-bis(4-substituted phenyl)-1methylpiperidin-4- ylidene] amino $\}$ - 2-thioxo-imidazolidin-4-one derivatives (3a3f) have been synthesized by the cyclization of compound (2a-2f), ethyl chloro acetate and fused sodium acetate. The chemical structures were confirmed by IR, ${ }^{1} \mathrm{H}$ NMR and elemental analysis. The synthesized compounds were screened for their antimicrobial activity against four antibacterial and four antifungal organisms.
\end{abstract}

Keywords: 2,6-Diphenylpiperidine-4-one, 2-Thioxo-imidazolidin-4-one, Antimicrobial activity.

\section{Introduction}

Hydantoins [imidazolidin-2,4-dione and 2-thioxo-imidazolin-4-one] are belongs to heterocyclic compound, which has a wide range of biological and pharmacological properties such as antimicrobial activity (antifungal, antibacterial) ${ }^{1}$, antitumor ${ }^{2}$, antiinflammatry ${ }^{2}$, anti $\mathrm{HIV}^{3}$, anti-hypertensive ${ }^{4}$, hydantoin exhibits diverse biological activities, such as anticonvulsant ${ }^{5}$, antifungal activities ${ }^{6}$, antithyroidal ${ }^{7}$, antiviral ${ }^{8}$, anti $\mathrm{HIV}^{9}$, tuber culosis $^{10}$, anti arrhythmic ${ }^{11}$ and anti convulsant ${ }^{12}$.

Many piperidine derivatives are found possessing pharmacological activities like anaesthetic activity ${ }^{14}$ and antimicrobial activity ${ }^{15}$. 2-Thioxo-imidazolindin-4-one was prepared from the reaction of aromatic aldehydes and thiosemicarbazide to give alkyl thiosemicarbazone followed by cyclization with ethyl chloroacetate in the presence of fused sodium acetate ${ }^{13}$. Above method was followed in the present study, thiosemicarbazide react with 2,6-diphenyl piperidin-4-one to give 2,6-disubstituted phenyl-piperidine-4thiosemicarbazone followed by cyclization with ethyl chloro acetate and fused sodium acetate. 
Major role of this study is to synthesis and screen for antimicrobial activity of series of 3-\{[2,6-bis (4-substituted phenyl)-1-methylpiperidin-4-ylidene] amino \}-2-thioxo-imidazolidin-4-one (3a-3f).

\section{Experimental}

Melting points were recorded in open capillary tubes and are uncorrected. $\operatorname{IR}\left(\mathrm{cm}^{-1}\right)$ was recorded in $\mathrm{KBr}$ on a FT-IR shimadzu $8201 \mathrm{pc}\left(4000-400 \mathrm{~cm}^{-1}\right)$ and ${ }^{1} \mathrm{H}$ NMR and Elemental analysis $(\mathrm{C}, \mathrm{H}, \mathrm{N}$ and $\mathrm{S})$ was undertaken using an Elemental analyzer model vario EL III. The purity of the compounds was checked by thin layer chromatography (TLC) with silica gel plates.

General procedure for synthesis of 1-methyl-2,6-diphenylpiperidin-4thiosemicarbazone (2a-2f)

A mixture of 1-methyl-2,6-disubstituted phenyl-piperidin-4-one (0.01 mol) and thiosemicarbazide $(0.01 \mathrm{~mol})$ in ethanol was heated under reflux for $6 \mathrm{~h}$. The reaction mixture was cooled and poured into crushed ice. The resulting solid produced was filtered, dried and recrystallized from suitable alcohols (Scheme 1).
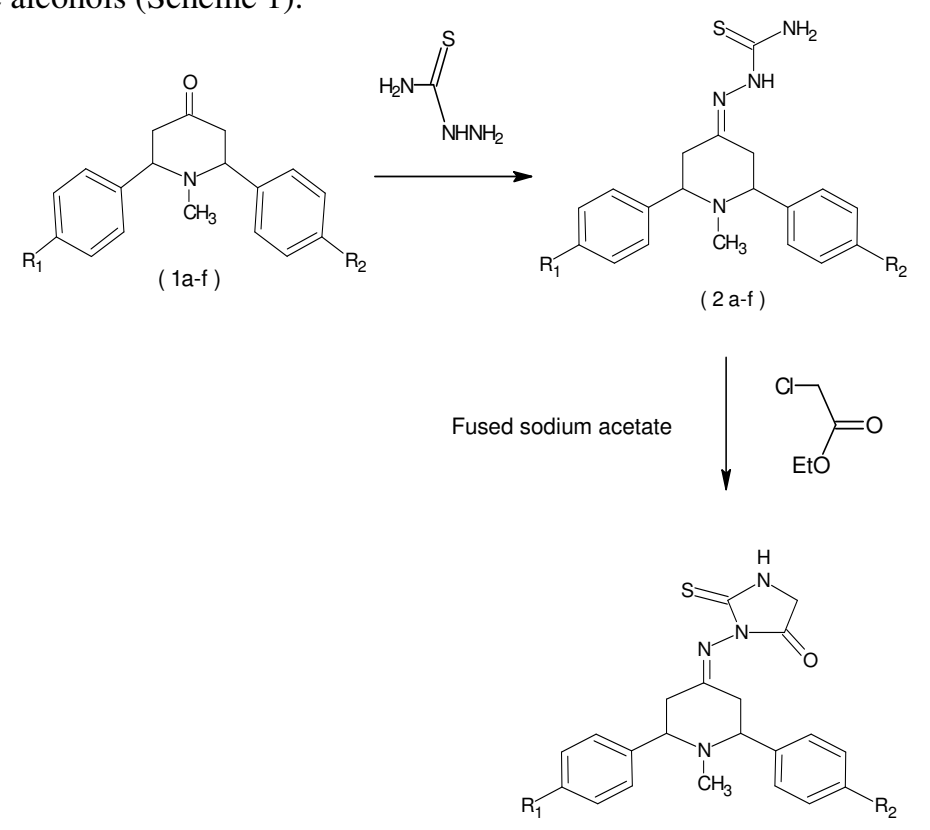

(3a-f)

Scheme 1. Synthesis of 4-thioxo-imidazolidin-2-one derivatives

\section{1-Methyl-2,6-diphenylpiperidin-4-thiosemicarbazone (2a)}

$\operatorname{IR}\left(\mathrm{KBr}, \mathrm{cm}^{-1}\right):$ 3475( $\left.\mathrm{NH}_{2}\right), 3060(\mathrm{Ar}-\mathrm{CH} \mathrm{str}), 2960(\mathrm{NH}), 1616(\mathrm{C}=\mathrm{N}), 1495(\mathrm{C}=\mathrm{S}), 727(\mathrm{~N}-\mathrm{C}-$ $\mathrm{N})$; ${ }^{1} \mathrm{H}$ NMR-(DMSO-d6, $\left.\delta(\mathrm{ppm})\right): 11.01 \quad(\mathrm{~s}, 1 \mathrm{H},=\mathrm{N}-\mathrm{NH}), \quad 7.23-6.56 \quad(\mathrm{~m}, 4 \mathrm{H}, \mathrm{Ar}-\mathrm{H})$, 6.73(s,1H, $\left.\mathrm{NH}_{2}\right), 3.74\left(\mathrm{~s}, 2 \mathrm{H}, 2,6-\mathrm{H}\right.$ in pipridine ring), $2.26\left(\mathrm{~s}, 3 \mathrm{H}, \mathrm{N}-\mathrm{CH}_{3}\right), 2.17(\mathrm{~s}, 2 \mathrm{H}, 3,5-\mathrm{H}$ in pipridine ring); Elemental analysis: Calculated for $\mathrm{C}_{19} \mathrm{H}_{22} \mathrm{~N}_{4} \mathrm{~S}$ : C,67.36; H,6.50; N,16.54; S, 9.45; Found: C, 67.34; H, 6.57; N, 16.52; S, 9.48\%.

\section{2,6-Bis(4-chlorophenyl)-1-methylpiperidin-4-thiosemicarbazone (2b)}

$\operatorname{IR}\left(\mathrm{KBr}, \mathrm{cm}^{-1}\right)$ : 3432( $\left.\mathrm{NH}_{2}\right)$, 3022(Ar-Ch Str), 2961(NH), 1632(C=N), 1423(C=S), 1082(N$\mathrm{C}-\mathrm{N}), 811(\mathrm{Ar}-\mathrm{Cl})$; ${ }^{1} \mathrm{H}$ NMR-(DMSO-d6, $\left.\delta(\mathrm{ppm})\right): 11.21(\mathrm{~s}, 1 \mathrm{H},=\mathrm{N}-\mathrm{NH}), 7.81-7.20$ (m,4H,Ar-H), 
7.15(s,2H, $\left.\mathrm{NH}_{2}\right), 3.68$ (s, 2H, 2,6-H in pipridine ring), $2.18\left(\mathrm{~s}, 3 \mathrm{H}, \mathrm{N}-\mathrm{CH}_{3}\right), 2.14(\mathrm{~s}, 2 \mathrm{H}, 3,5-\mathrm{H}$ in pipridine ring); Elemental analysis: Calculated for $\mathrm{C}_{19} \mathrm{H}_{20} \mathrm{Cl}_{2} \mathrm{~N}_{4} \mathrm{~S}: \mathrm{C}$, 55.97; H,4.90;N,13.74;S,7.85 ; Found: C, 55.94; H, 4.87; N, 13.71 S, 7.81\%.

\section{2, 6-Bis (4-hydroxy phenyl)-1-methylpiperidin-4-one thiosemicarbazone (2c)}

$\mathrm{IR}\left(\mathrm{KBr}, \mathrm{cm}^{-1}\right)$ : 3473( $\left.\mathrm{NH}_{2}\right), 3121$ ( Ar-CH Str), $2931(\mathrm{NH}), 1626(\mathrm{C}=\mathrm{N}), 1450(\mathrm{Ar}-\mathrm{OH}$ str), 1136 $(\mathrm{C}=\mathrm{S}), 1122(\mathrm{~N}-\mathrm{C}-\mathrm{N})$; ${ }^{1} \mathrm{H}$ NMR- $(\mathrm{DMSO}-\mathrm{d} 6, \delta(\mathrm{ppm})): 11.96(\mathrm{~s}, 1 \mathrm{H}, \mathrm{Ar}-\mathrm{OH}), 10.91(\mathrm{~s}, 1 \mathrm{H},=\mathrm{N}-$ $\mathrm{NH}), 7.51-7.34(\mathrm{~m}, 4 \mathrm{H}, \mathrm{Ar}-\mathrm{H}), 7.20\left(\mathrm{~s}, 2 \mathrm{H}, \mathrm{NH}_{2}\right), 3.54(\mathrm{~s}, 2 \mathrm{H}, 2,6-\mathrm{H}$ in pipridine ring) $2.28(\mathrm{~s}, 3 \mathrm{H}$, $\left.\mathrm{N}-\mathrm{CH}_{3}\right), 2.20\left(\mathrm{~s}, 2 \mathrm{H}, 3,5-\mathrm{H}\right.$ in pipridine ring); Elemental analysis: Calculated for $\mathrm{C}_{19} \mathrm{H}_{22} \mathrm{~N}_{4} \mathrm{O}_{2} \mathrm{~S}$ : C,33.12; H,5.01; N,14.05; S,8.02; Found: C,33.09; H,5.04;N,14.02; S,8.04\%.

\section{2,6-Bis(4- methoxyphenyl)-1-methylpiperidin-4-one thiosemicarbazone (2d)}

$\operatorname{IR}\left(\mathrm{KBr}, \mathrm{cm}^{-1}\right)$ : 3465( $\left.\mathrm{NH}_{2}\right), 3145(\mathrm{Ar}-\mathrm{H}), 2902(\mathrm{NH}), 1675(\mathrm{C}=\mathrm{N}), 1138(\mathrm{C}=\mathrm{S}), 1022(\mathrm{~N}-\mathrm{C}-\mathrm{N})$; ${ }^{1} \mathrm{H}$ NMR-(DMSO-d6, $\left.\delta(\mathrm{ppm})\right): 11.12(\mathrm{~s}, 1 \mathrm{H},=\mathrm{N}-\mathrm{NH}), 7.61-7.49(\mathrm{~m}, 4 \mathrm{H}, \mathrm{Ar}-\mathrm{H}), 7.30$ $\left(\mathrm{s}, 2 \mathrm{H}, \mathrm{NH}_{2}\right), 4.12\left(\mathrm{~s}, 3 \mathrm{H}, \mathrm{Ar}-\mathrm{CH}_{3} \mathrm{O}\right), 3.54(\mathrm{~s}, 2 \mathrm{H}, 2,6-\mathrm{H}$ in pipridine ring) $2.39(\mathrm{~s}, 3 \mathrm{H}$, $\left.N-\mathrm{CH}_{3}\right) ; 2.20\left(\mathrm{~s}, 2 \mathrm{H}, 3,5-\mathrm{H}\right.$ in pipridine ring); Elemental analysis: Calculated for $\mathrm{C}_{19} \mathrm{H}_{22} \mathrm{~N}_{4} \mathrm{~S}$ : C,63.36; H,6.53; N,14.57; S,8.44; Found: C,63.34; H,6.50; N,14.50; S,8.48\%.

\section{2,6-Bis(4-nitro phenyl)-1-methylpiperidin-4-one thiosemicarbazone (2e)}

$\operatorname{IR}\left(\mathrm{KBr}, \mathrm{cm}^{-1}\right): 3445\left(\mathrm{NH}_{2}\right), 3092(\mathrm{Ar}-\mathrm{H}), 2960(\mathrm{NH}), 1617(\mathrm{C}=\mathrm{N}), 1550\left(\mathrm{Ar}-\mathrm{NO}_{2}\right), 1131(\mathrm{C}=\mathrm{S})$, 1042(N-C-N); ${ }^{1} \mathrm{H}$ NMR-(DMSO-d6, $\delta$ (ppm)):11.24(s, 1H, =N-NH ), 7.59-7.49(m,4H,Ar-H), $7.32\left(\mathrm{~s}, 2 \mathrm{H}, \mathrm{NH}_{2}\right), 3.45\left(\mathrm{~s}, 2 \mathrm{H}, 2,6-\mathrm{H}\right.$ in pipridine ring), $2.23\left(\mathrm{~s}, 3 \mathrm{H}, \mathrm{N}-\mathrm{CH}_{3}\right) ; 2.11(\mathrm{~s}, 2 \mathrm{H}, 3,5-\mathrm{H}$ in pipridine ring); Elemental analysis: Calculated for $\mathrm{C}_{19} \mathrm{H}_{20} \mathrm{~N}_{6} \mathrm{O}_{4} \mathrm{~S}$ : C,53.21; H,4.66; N,19.60; S,7.46 ; Found: C, 53.27; H, 4.63; N, 19.57; S, 7.42\%.

\section{2,6-Bis(4-dimethyl amine phenyl)-1-methylpiperidin-4-one thiosemicarbazone (2f)} $\operatorname{IR}\left(\mathrm{KBr}, \mathrm{cm}^{-1}\right): 3388\left(\mathrm{NH}_{2}\right), 3112(\mathrm{Ar}-\mathrm{H}), 2927(\mathrm{NH}), 1676(\mathrm{C}=\mathrm{N}), 1127(\mathrm{C}=\mathrm{S}), 1052(\mathrm{~N}-\mathrm{C}-$ $\mathrm{N})$; ${ }^{1} \mathrm{H}$ NMR- (DMSO-d6, $\left.\delta(\mathrm{ppm})\right): 11.30(\mathrm{~s}, 1 \mathrm{H},=\mathrm{N}-\mathrm{NH}), \quad 7.51-7.47(\mathrm{~m}, 4 \mathrm{H}, \mathrm{Ar}-\mathrm{H}), 7.39$ $\left(\mathrm{s}, 2 \mathrm{H}, \mathrm{NH}_{2}\right), \quad 2.72\left(\mathrm{~s}, 6 \mathrm{H}, \mathrm{Ar}-\mathrm{N}\left(\mathrm{CH}_{3}\right)_{2}\right), 3.88(\mathrm{~s}, 2 \mathrm{H}, 2,6-\mathrm{H}$ in pipridine ring), $2.28(\mathrm{~s}, 3 \mathrm{H}, \mathrm{N}-$ $\mathrm{CH}_{3}$ ); 2.17(s,2H, 3,5-H in pipridine ring); Elemental analysis: Calculated for $\mathrm{C}_{23} \mathrm{H}_{32} \mathrm{~N}_{6} \mathrm{~S}$ : C,65.00; H,7.53;N,19.78;S,7.53; Found: C, 65.05; H, 7.57; N, 19.75; S, 7.55\%.

\section{General procedure for synthesis of 3-[(1-methyl-2, 6-diphenylpiperidin-4-ylidene)} amino]-2-thioxo-imidazolidin-4-one (3a-3f)

A mixture of compound 3-[(1-Methyl-2,6-disubstituted phenylpiperidin-4-ylidene)amino]-2thioxo-imidazolidin-4-one (2a-2f) $(0.1 \mathrm{~mol})$, ethyl chloroacetate $(0.1 \mathrm{~mol})$ and fused sodium acetate $(0.1$ mole $)$ in ethanol, the mixture was heated under reflux for $6 \mathrm{~h}$. The reaction mixture was cooled and poured into crushed ice. The resulting solid product was filtered, dried and recryatallized from absolute ethanol (Table 1).

Table 1. Characterization data of the compounds (3a-3f)

\begin{tabular}{ccccc}
\hline Compd.no & $\mathrm{R} 1$ & $\mathrm{R} 2$ & $\mathrm{~m} . \mathrm{p}{ }^{\circ} \mathrm{C}$ & Yield \% \\
\hline 3a & $-\mathrm{H}$ & $-\mathrm{H}$ & 231 & 54 \\
3b & $-\mathrm{Cl}$ & $-\mathrm{Cl}$ & 240 & 59 \\
3c & $-\mathrm{OH}$ & $-\mathrm{OH}$ & 252 & 57 \\
3d & $-\mathrm{OCH}_{3}$ & $-\mathrm{OCH}_{3}$ & 211 & 51 \\
3e & $-\mathrm{NO}_{2}$ & $-\mathrm{NO}_{2}$ & 227 & 48 \\
3f & $-\mathrm{N}_{2}\left(\mathrm{CH}_{3}\right)_{2}$ & $-\mathrm{N}^{2}\left(\mathrm{CH}_{3}\right)_{2}$ & 238 & 57 \\
\hline
\end{tabular}


3-[(1-Methyl-2, 6-diphenylpiperidin-4-ylidene) amino]-2-thioxo-imidazolidin-4-one (3a) $\mathrm{IR}\left(\mathrm{KBr} \mathrm{cm}^{-1}\right)$ : 3084(NH), 1722(C=O), $1664(\mathrm{C}=\mathrm{N}), 1495(\mathrm{C}=\mathrm{S})$; ${ }^{1} \mathrm{H}$ NMR -(DMSO-d6, $\delta(\mathrm{ppm})$ ): $\delta 10.32(\mathrm{~s}, 1 \mathrm{H} \mathrm{NH}), 7.58-7.21(\mathrm{~m}, 4 \mathrm{H}, \mathrm{Ar}-\mathrm{H}), 3.90\left(\mathrm{~s}, 2 \mathrm{H}, 5 \mathrm{CH}_{2}\right), 3.54(\mathrm{~s}, 2 \mathrm{H}, 2,6-\mathrm{H}$ in pipridine ring), 2.41(s,2H,3,5-H in pipridine ring) $2.18\left(\mathrm{~s}, 3 \mathrm{H}, \mathrm{N}-\mathrm{CH}_{3}\right)$, Elemental analysis: Calculated for $\mathrm{C}_{21} \mathrm{H}_{22} \mathrm{~N}_{4} \mathrm{OS}$ : C,66.58; H,5.81;N,14.79;S,16.90; Found: C, 66.55; H, 5.79; N, 14.75; S, $16.88 \%$.

3-\{[2,6-Bis(4-chlorophenyl)-1-methylpiperidin-4-ylidene]amino\}-2-thioxo-imidazolidin-4one $(3 \mathrm{~b})$

$\operatorname{IR}\left(\mathrm{KBr}, \mathrm{cm}^{-1}\right): 3021(\mathrm{NH}), 1723(\mathrm{C}=\mathrm{O}), 1666(\mathrm{C}=\mathrm{N}), 1520(\mathrm{C}=\mathrm{S}), 751(\mathrm{Ar}-\mathrm{Cl}) ;{ }^{1} \mathrm{H}$ NMR$(\mathrm{DMSO}-\mathrm{d} 6, \delta(\mathrm{ppm})): \delta 10.23(\mathrm{~s}, 1 \mathrm{H} \mathrm{NH}), 7.58-7.31(\mathrm{~m}, 4 \mathrm{H}, \mathrm{Ar}-\mathrm{H}), 3.42\left(\mathrm{~s}, 2 \mathrm{H}, 5 \mathrm{CH}_{2}\right), 3.54(\mathrm{~s}$, $2 \mathrm{H}, 2,6-\mathrm{H}$ in pipridine ring), 2.41( $\mathrm{s}, 2 \mathrm{H}, 3,5-\mathrm{H}$ in pipridine ring), $2.12\left(\mathrm{~s}, 3 \mathrm{H}, \mathrm{N}_{-} \mathrm{CH}_{3}\right)$; Elemental analysis: Calculated for For $\mathrm{C}_{21} \mathrm{H}_{20} \mathrm{Cl}_{2} \mathrm{~N}_{4} \mathrm{OS}$ : C,56.32; H,4.47; N,12.51;S,7.15; Found: C,56.30; H,4.42; N,12.54; S,7.12\%.

3-\{[2,6-Bis(4-hydroxy phenyl)-1-methylpiperidin-4-ylidene]amino\}-2-thioxo-imidazolidin4-one $(\mathbf{3 c})$

$\mathrm{IR}\left(\mathrm{KBr}, \mathrm{cm}^{-1}\right):$ 3032(NH), 1722(C=O), $1664(\mathrm{C}=\mathrm{N}), 1459(\mathrm{Ar}-\mathrm{OH}), 1432(\mathrm{C}=\mathrm{S}) ;{ }^{1} \mathrm{H}$ NMR(DMSO-d6, $\delta$ (ppm) ): $\delta 11.21(\mathrm{~s}, 1 \mathrm{H}, \mathrm{Ar}-\mathrm{OH}), \delta 10.11(\mathrm{~s}, 1 \mathrm{H}, \mathrm{NH}), 7.58-7.11(\mathrm{~m}, 4 \mathrm{H}, \mathrm{Ar}-\mathrm{H})$, $4.21\left(\mathrm{~s}, 2 \mathrm{H}, 5 \mathrm{CH}_{2}\right), 3.54(\mathrm{~s}, 2 \mathrm{H}, 2,6-\mathrm{H}$ in pipridine ring $), 2.20\left(\mathrm{~s}, 3 \mathrm{H}, \mathrm{N}-\mathrm{CH}_{3}\right) ; 2.41(\mathrm{~s}, 2 \mathrm{H}, 3,5-$ $\mathrm{H}$ in pipridine ring), $2.20\left(\mathrm{~s}, 3 \mathrm{H}, \mathrm{N}-\mathrm{CH}_{3}\right)$; Elemental analysis: Calculated for $\mathrm{C}_{21} \mathrm{H}_{22} \mathrm{~N}_{4} \mathrm{O}_{3} \mathrm{~S}: \mathrm{C}$, 61.39; H,5.35;N,13.64;S,7.79; Found: C,61.28; H,5.32; N,13.61; S,7.75\%.

3-\{[2,6-Bis(4-methoxy phenyl)-1-methylpiperidin-4-ylidene]amino\}-2-thioxo-imidazolidin4-one (3d)

$\operatorname{IR}\left(\mathrm{KBr}, \mathrm{cm}^{-1}\right)$ : 3051(NH), 1783(C=O), $1675(\mathrm{C}=\mathrm{N}), 1432(\mathrm{C}=\mathrm{S}) ;{ }^{1} \mathrm{H}$ NMR-(DMSO-d6, $\delta$ (ppm) ): $\delta 10.67(\mathrm{~s}, 1 \mathrm{H} \mathrm{NH}), 7.44-7.21(\mathrm{~m}, 4 \mathrm{H}, \mathrm{Ar}-\mathrm{H}), 4.12\left(\mathrm{~s}, 3 \mathrm{H}, \mathrm{Ar}-\mathrm{OCH}_{3}\right), 4.07\left(\mathrm{~s}, 2 \mathrm{H}, \mathrm{CH}_{2}\right)$, $3.54(\mathrm{~s}, 2 \mathrm{H}, 2,6-\mathrm{H}$ in pipridine ring $), 2.48\left(\mathrm{~s}, 3 \mathrm{H}, \mathrm{N}-\mathrm{CH}_{3}\right) ; 2.41(\mathrm{~s}, 2 \mathrm{H}, 3,5-\mathrm{H}$ in pipridinering); Elemental analysis: Calculated for $\mathrm{C}_{23} \mathrm{H}_{26} \mathrm{~N}_{6} \mathrm{O}_{3} \mathrm{~S}: \mathrm{C}, 62.93$; H,5.92;N,19.15;S,7.29; Found: C,62.90; H,5.93; N,19.12; S,7.25\%.

3-\{[2,6-Bis(4-nitro phenyl)-1-methylpiperidin-4-ylidene]amino\}-2-thioxo-imidazolidin-4one (3e)

$\operatorname{IR}\left(\mathrm{KBr}, \mathrm{cm}^{-1}\right): 3014(\mathrm{NH}), 1742(\mathrm{C}=\mathrm{O}), 1674(\mathrm{C}=\mathrm{N}), 1553\left(\mathrm{Ar}-\mathrm{NO}_{2}\right), 1438(\mathrm{C}=\mathrm{S}) ;{ }^{1} \mathrm{H}$ NMR (DMSO-d6, $\delta$ (ppm)): $\delta 10.29(\mathrm{~s}, 1 \mathrm{H} \mathrm{NH}), 7.28-6.97(\mathrm{~m}, 4 \mathrm{H}, \mathrm{Ar}-\mathrm{H}), 3.97\left(\mathrm{~s}, 2 \mathrm{H}, 5 \mathrm{CH}_{2}\right), 2.14$ $\left(\mathrm{s}, 3 \mathrm{H}, \mathrm{N}-\mathrm{CH}_{3}\right) ; 2.41(\mathrm{~s}, 2 \mathrm{H}, 3,5-\mathrm{H}$ in pipridine ring), $3.54(\mathrm{~s}, 2 \mathrm{H}, 2,6-\mathrm{H}$ in pipridine ring); Elemental analysis: Calculated for $\mathrm{C}_{21} \mathrm{H}_{20} \mathrm{~N}_{6} \mathrm{O}_{5} \mathrm{~S}: \mathrm{C}, 53.79 ; \mathrm{H}, 4.26 ; \mathrm{N}, 17.93 ; \mathrm{S}, 6.83$; Found: $\mathrm{C}$, $53.75 ; \mathrm{H}, 4.21 ; \mathrm{N}, 17.90 \mathrm{~S}, 6.80 \%$.

3-\{[2,6-Bis(4-dimethylamine phenyl)-1-methylpiperidin-4-ylidene]amino\}-2thioxo-imidazolidin-4-one (3f)

$\mathrm{IR}\left(\mathrm{KBr}, \mathrm{cm}^{-1}\right)$ : 3045(NH), 1736(C=O), $1652(\mathrm{C}=\mathrm{N}), 1437(\mathrm{C}=\mathrm{S}) ;{ }^{1} \mathrm{H}$ NMR(DMSO-d6, $\delta(\mathrm{ppm})): 10.69(\mathrm{~s}, 1 \mathrm{H} \mathrm{NH}), 7.42-7.30(\mathrm{~m}, 4 \mathrm{H}, \mathrm{Ar}-\mathrm{H}), 3.72\left(\mathrm{~s}, 2 \mathrm{H}, 5 \mathrm{CH}_{2}\right), 3.54(\mathrm{~s}, 2 \mathrm{H}, 2,6-\mathrm{H}$ in pipridine ring), 2.79(s,6H, $\left.\mathrm{Ar}-\mathrm{N}\left(\mathrm{CH}_{3}\right)_{2}\right), 2.41(\mathrm{~s}, 2 \mathrm{H}, 3,5-\mathrm{H}$ in pipridine ring), 2.28 (s, $3 \mathrm{H}$, $\mathrm{N}-\mathrm{CH}_{3}$ ); Elemental analysis: Calculated for $\mathrm{C}_{25} \mathrm{H}_{32} \mathrm{~N}_{6} \mathrm{OS}: \mathrm{C}, 64.56 ; \mathrm{H}, 6.88 ; \mathrm{N}, 18.07 ; \mathrm{S}, 6.88$; Found: C, 64.54; H, 6.85; N, 18.02; S, 6.82\%.

\section{Results and Discussion}

The IR spectrum of compounds (2a-2f), show an absorption band at 3488-3432, 1676-1616, 1138$1127 \mathrm{~cm}^{-1}$ due to corresponding to the $\mathrm{NH}_{2}, \mathrm{C}=\mathrm{N}$ and $\mathrm{C}=\mathrm{O}$ groups respectively. The IR spectrum 
of compounds (3a-3f), show an absorption band at 3014-3084, 1783-1722, 1652-1675 and $1432-1520 \mathrm{~cm}^{-1}$ due to corresponding to the $\mathrm{NH}, \mathrm{C}=\mathrm{O}, \mathrm{C}=\mathrm{N}$ and $\mathrm{C}=\mathrm{S}$ groups respectively.

The ${ }^{1} \mathrm{H}$ NMR spectrum of compounds (2a-2f), show a singlet at $\delta 11.30-10.91 \mathrm{ppm}$ attributable to $\mathrm{C}=\mathrm{N}-\mathrm{NH}$ protons. The compounds (3a-3f) have very important signal at $\delta$ 10.69-10.11 and 4.27 -3.97 ppm corresponding to the $\mathrm{NH}, \mathrm{CH}_{2} \mathrm{~N}$ protons respectively.

\section{Antimicrobial activity}

\section{In vitro antibacterial screening}

The compounds (3a-3f) were evaluated for their in vitro antibacterial activity against Staphylococcus aureus (MTCC-96), Enterococcus faecalis (MTCC-439), Escherchia coli (MTCC-739), Pseudomonas aeruginosa (MTCC-2453) by agar dilution method ${ }^{18,19}$ was performed using Mueller -Hinton agar (Hi-Media) medium. Each compound was tested at a concentration of $100 \mu \mathrm{g} / \mathrm{mL}$ in DMSO. The zone of inhibition was measured after $24 \mathrm{~h}$ incubation at $37{ }^{\circ} \mathrm{C}$. Inhibition zone of the compounds clearly indicate the (3f) compound was highly active against S.aureus. The results are presented in Table 2. Inhibition of the compounds (3a-3f) were clearly observed in $\mathrm{NO}_{2}$ substituted at benzaldehyde containing compound (3e) showed maximum antibacterial potency. Compound (3c) has nearly activity against Staphylococcus aureus, Enterococcus faecalis and compound (3d) has less activity compared with norfloxacin against all bacterial organisms.

Table 2. Antibacterial activity of the compounds (3a-3f)

\begin{tabular}{ccccc}
\hline Compounds & S. aureus & E.faecalis & E. coli & P. aeruginosa \\
\hline 3a & 20 & 14 & 16 & 13 \\
3b & 19 & 12 & 20 & 16 \\
3c & 21 & 13 & 20 & 8 \\
3d & 12 & - & 18 & - \\
3e & 22 & 13 & 21 & 15 \\
3f & 23 & 8 & 13 & 10 \\
Standard & 22 & 15 & 24 & 21
\end{tabular}

Zone of the inhibition measured in $(\mathrm{mm})$. Indicate bacteria's are resistant to the compound $10 \mu \mathrm{g} / \mathrm{mL}$ Norfloxacin is used as the standard drug $100 \mu \mathrm{g} / \mathrm{mL}$

\section{In vitro antifungal screening}

The compounds (3a-3f) were evaluated for their in vitro antifungal activity against Aspergillus niger, Candia albicans, Aspergillus fumigatus and Cryptococcus neoformans using an agar dilution method ${ }^{20}$ with sabouraud's dextrose agar (Hi-Media). Each compound was tested at a concentration of $100 \mu \mathrm{g} / \mathrm{mL}$ in DMSO. The zone of inhibition was measured after incubating at $37{ }^{\circ} \mathrm{C}$ for $24 \mathrm{~h}$. It was evident from screening results that only 4-OH phenyl substituted compound (3c) has remarkably enhanced the antifungal action. When compared to ketoconazole, compound (3b) is moderately active against all antifungal organisms. Compounds (3a, 3d, 3e and 3f) are less active against all antifungal organisms. All compounds are compared with ketoconazole the results are presented in Table 3.

\section{Conclusion}

The series of compound (3a-3f) 2-thioxo-imidazolidin-4-one derivatives were synthesized and screened for their antimicrobial activity. The compound (3f) has highly anti bacterial activity against S.aureus compared with norfloxacin standard and compound (3c) has highly antifungal activity aganist $A$. niger and A.fumigates compared with a ketoconazole standard. 
Table 3. Antifungal activity of the compounds (3a-3f)

\begin{tabular}{ccccc}
\hline Compounds & A. niger, & C.albicans & A.fumigatus & Cr.neoformans \\
\hline 3a & 12 & 10 & 12 & 13 \\
3b & 19 & 19 & 14 & 16 \\
3c & 24 & 23 & 18 & 14 \\
3d & 13 & 12 & 16 & 14 \\
3e & 8 & 10 & 12 & 8 \\
3f & 12 & 8 & - & - \\
Standard & 22 & 24 & 16 & 18 \\
\hline
\end{tabular}

Zone of the inhibition measured in (mm). Indicate fun gals are resistant to the compound $100 \mu \mathrm{g} / \mathrm{mL}$ Ketoconazole is used as the standard $100 \mu \mathrm{g} / \mathrm{mL}$

\section{Acknowledgment}

We wish to thank the state Government, for providing state government fellowship and extending financial support. We wish to thank one of the authors Dr. J. Selvin, Department of Microbiology, Bharathidasan University, for his help in microbial activities. We sincerely thank Dr.M. Sheik Mohamed, Principal Jamal Mohamed College, for providing Laboratory facilities.

\section{References}

1. Martan J, Enisz J, Hosztafi S and Timer T, J Agri food Chem., 1993, 41, 148-152.

2. Ahmed K I, Carbohydr Res., 1998, 306, 567.

3. Comber R N, Reynolds R C, Friedeich J D, Magaikian R A, Buckheit R W, Truss J S, Shannon W M and Secrist J A, J Med Chem., 1992, 35, 3567-3572.

4. Menendaz J C, Diaz M P, Bellelverl C and Sollhuber M M, Eur J Med Chem., 1992, 27, 66.

5. Noveli A, Anales Farm Y, Bioquim, 1945, 21, 81, (Chem Abstr., 1956, 50, 4922).

6. Cremlyn R J, Swin bourne F J, Shode O O and Lynch J, J Heterocyclic Chem., 1987, 24, 117-121.

7. MarX J V, Richert D A and Westerfeld W W, J Med Chem., 1970, 13, 1179-1181.

8. El-Barbary A A, Khodair A I, Pedersan E B and Nielsen C, J Med Chem., 1994, 37, 73.

9. Cherouvrier J R, Carreaux F and Bazureau J P, Molecules, 2004, 9, 867-875.

10. Arches S, Unser M J and Froelich E, J Am Chem Soc., 1956, 78, 6182.

11. Hevera H J and Strycker W G, Chem Absr., 1997, 86, 106586.

12. Cortes S, LIas Z K, Watson D and Kohn H, J Med Chem., 1985, 28, 601-606.

13. Abd M E and Fatlah E I, Indian J Chem., 2006, 45B, 2523-2533.

14. Perumal R V, Adiraj M and Shanmugapandiyan P, Indian Drugs, 2001, 38, 156-159.

15. Mobio I G, Soldatenkov A T, Federov V O, Ageev E A, Sergeeva N D, Lin S, Stashenko E E, Prostakov N S and Andreeva E I, Khim Farm Zh., 1989, 23, 421-427.

16. Noller C R and Baliah V, J Am Chem Soc., 1948, 70, 3853.

17. Natesh Rameshkumar, Anantharaman Veena, Raju Ilavarason, Mandalees Waran Adiraj, Pitchaimuthu Shanmugapandiyan and Seshaiah Krishnan Sridhar, Biol Pharm Bull., 2003, 26(2), 188-193.

18. Bauer A W, Kirby W M, Sherris J C and Turck M, Am J Clin Pathol, 1966, 39(5), 493-496.

19. Robert G Petersdorf and John C Sherris, Am J Med., 1965, 39(5), 766-779.

20. Gillespie S H, Medical Microbiology-Illustrated, Butterworth Heinemann: London, 1994, 234-247. 


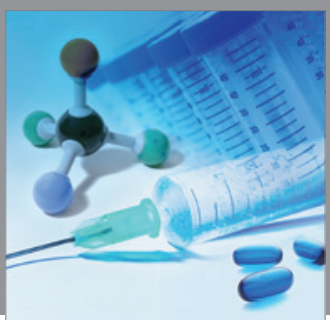

International Journal of

Medicinal Chemistry

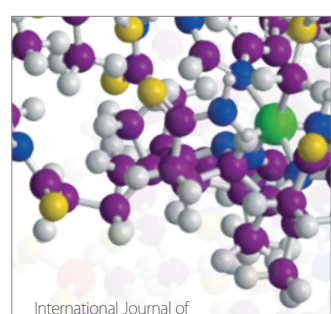

Carbohydrate Chemistry

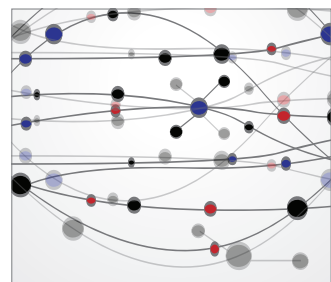

The Scientific World Journal
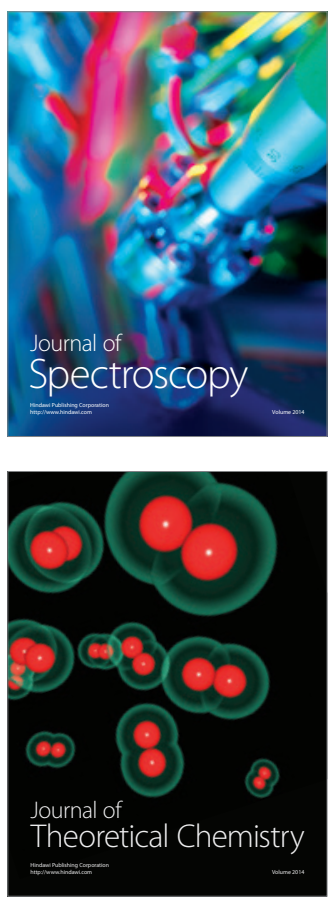
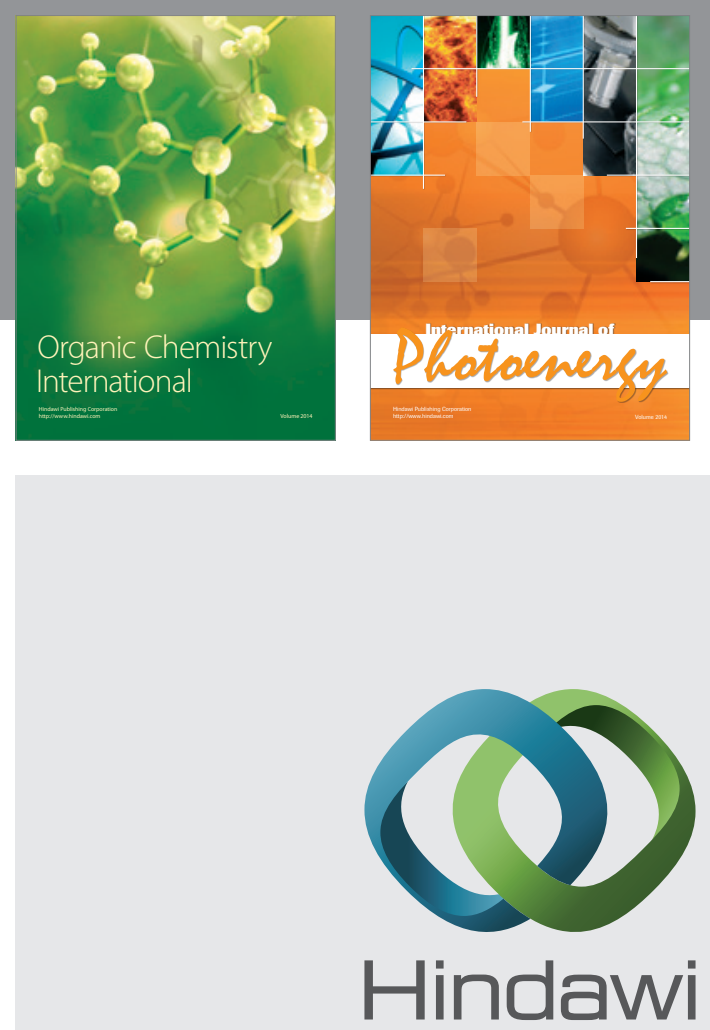

Submit your manuscripts at

http://www.hindawi.com
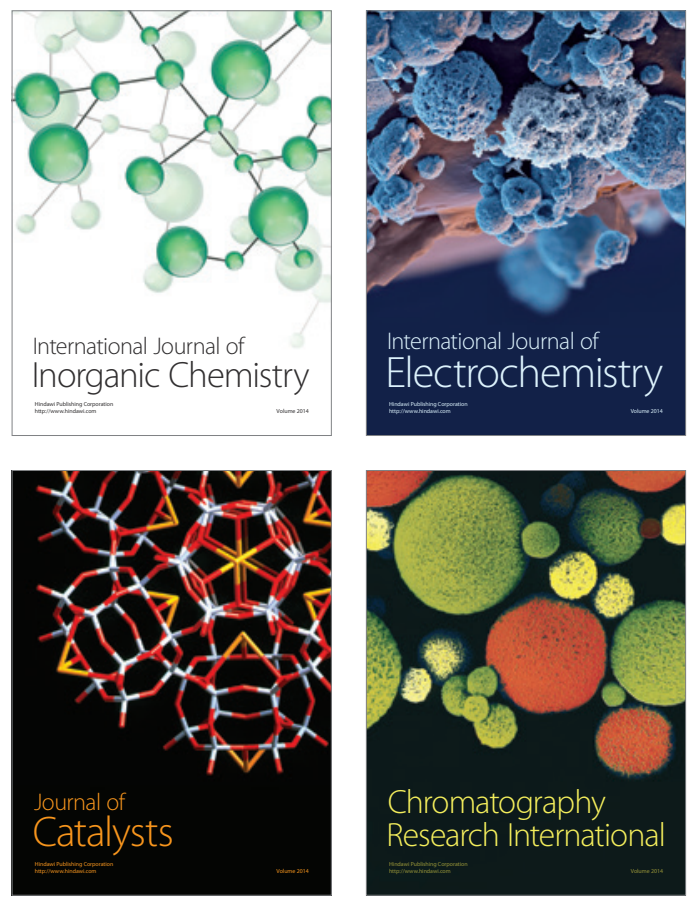
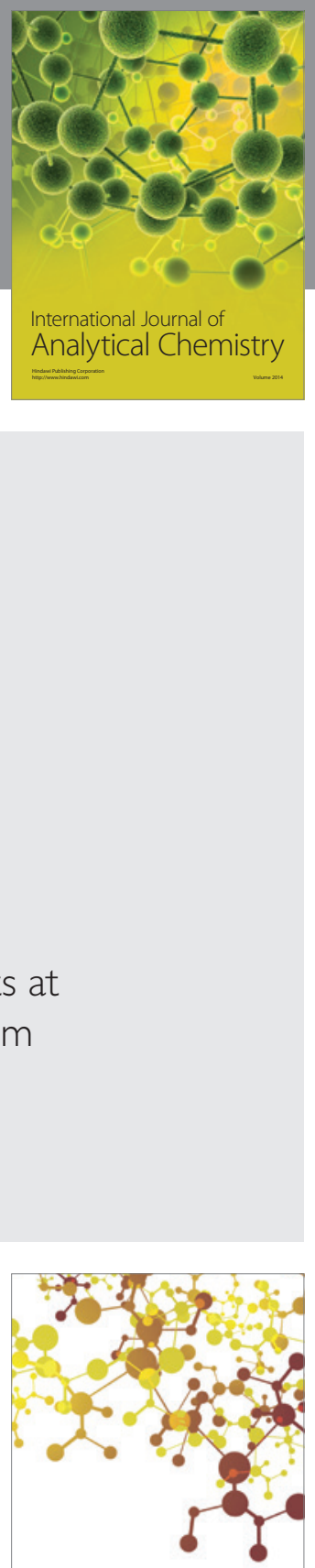

Journal of

Applied Chemistry
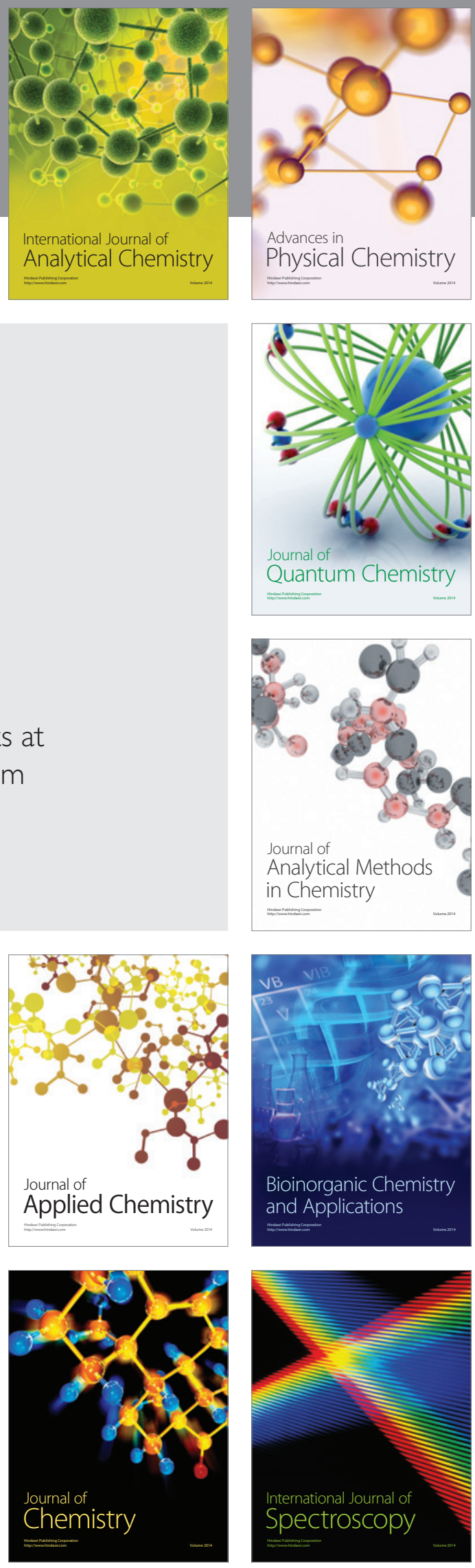